\title{
Accelerated Hypofractionation for Elderly or Frail Patients with a Newly Diagnosed Glioblastoma Improves Survival
}

Haley Kopp Perlow ( $\nabla$ haley.perlow@osumc.edu )

Ohio State University https://orcid.org/0000-0001-8667-0782

\section{Alexander Yaney}

Ohio State University Science and Engineering Library: The Ohio State University

Michael Yang

Ohio State University

\section{Brett Klamer}

Ohio State University

Jennifer Matsui

Ohio State University

\section{Raju R Raval}

Ohio State University

\section{Dukagjin M. Blakaj}

Ohio State University

\section{Andrea Arnett}

Ohio State University

\section{Sasha Beyer}

Ohio State University

James B. Elder

Ohio State University Science and Engineering Library: The Ohio State University

Mario Ammirati

Ohio State University

\section{Russell Lonser}

Ohio State University

Douglas Hardesty

Ohio State University

\section{Shirley Ong}

Ohio State University

\section{Pierre Giglio}

Ohio State University

Clement Pillainayagam 
Ohio State University

\section{Justin Goranovich}

Ohio State University

John Grecula

Ohio State University

\section{Arnab Chakravarti}

Ohio State University

\section{Vinai Gondi}

Northwestern University

\section{Paul D. Brown}

Mayo Clinic

Joshua D. Palmer

Ohio State University

\section{Research Article}

Keywords: glioblastoma (GBM), temozolomide (TMZ), hypofractionated radiation.

Posted Date: October 25th, 2021

DOI: https://doi.org/10.21203/rs.3.rs-992179/v1

License: (c) (i) This work is licensed under a Creative Commons Attribution 4.0 International License. Read Full License 


\section{Abstract}

\section{Background:}

The standard of care for elderly glioblastoma (GBM) patients is 40 Gy in 15 fraction radiotherapy with temozolomide (TMZ). However, this regimen has a lower biologic equivalent dose (BED) compared to the Stupp regimen of $60 \mathrm{~Gy}$ in 30 fractions. We hypothesize that accelerated hypofractionated radiation of $52.5 \mathrm{~Gy}$ in 15 fractions (BED equivalent to Stupp) will have superior survival compared to 40 Gy in 15 fractions.

\section{Methods:}

Elderly patients ( $\geq 65$ years old) who received hypofractionated radiation with TMZ from 2010-2020 were included in this analysis. Overall survival (OS) and progression free survival (PFS) were defined as the time elapsed between surgery/biopsy and death from any cause or progression. Baseline characteristics were compared between patients who received $40 \mathrm{~Gy}$ and $52.5 \mathrm{~Gy}$. Univariable and multivariable analyses were performed.

\section{Results:}

Sixty-six newly diagnosed patients were eligible for analysis. Thirty-nine patients were treated with 40 Gy in 15 fractions while twenty-seven were treated with $52.5 \mathrm{~Gy}$ in 15 fractions. Patients had no significant differences in age, sex, methylation status, or performance status. Overall survival (OS) was superior in the 52.5 Gy group (14.1 months) when compared to the $40 \mathrm{~Gy}$ group (7.9 months, $p=0.011$ ). Isoeffective dosing to 52.5 Gy was shown to be an independent prognostic factor for improved OS on multivariable analysis.

\section{Conclusions:}

Isoeffective dosing to $52.5 \mathrm{~Gy}$ in 15 fractions was associated with superior OS compared to standard of care 40 Gy in 15 fractions. These hypothesis generating data support accelerated hypofractionation in future prospective trials.

\section{Introduction}

High-grade gliomas encompass approximately $70 \%$ of the approximately 23,000 malignant brain tumors diagnosed in the United States each year. A majority of these high-grade gliomas are glioblastoma (GBM) ${ }^{1}$. Maximal safe resection followed by adjuvant radiation therapy has proven to be superior to resection alone, with early randomized trials showing a doubling of overall survival (OS) with the addition of adjuvant radiation therapy ${ }^{2,3}$. A significant dose-effect relationship for these patients has been demonstrated, with a stepwise increase of radiation dose from 45 Gy to 60 Gy showing a significant improvement in OS at each interval ${ }^{4}$. This study evaluating the dose-effect relationship led to the current understanding that de-escalation of radiotherapy below $60 \mathrm{~Gy}$ portends inferior outcomes. As such, 
radiation therapy to a total of $60 \mathrm{~Gy}$ in 30 fractions became the preferred adjuvant therapy dose. The current standard of care for GBM patients including adjuvant radiation with concurrent and adjuvant temozolomide (TMZ) was then determined in the Stupp trial, which demonstrated a significant OS benefit (27.2\% versus $10.9 \%$ at 2 years) with the addition of concurrent and adjuvant TMZ compared to adjuvant radiotherapy alone ${ }^{5}$. However, even with this regimen outcomes remain poor with a median oS of 16 months ${ }^{1}$.

The median age at diagnosis of GBM is 64 years $^{6}$. In the Stupp trial, elderly patients ( $>60$ years old) had a median OS of 10.9-11.8 months ${ }^{5}$. However, even elderly patients have a survival benefit with radiation in comparison to supportive care alone ${ }^{7}$. Recent studies have suggested that hypofractionated deescalated radiation therapy to $40 \mathrm{~Gy}$ in 15 fractions could be a reasonable alternative for elderly patients or those with a poor performance status ${ }^{8-10}$. The IAEA trial including elderly (age $\geq 65$ ) or frail (Karnofsky Performance Status 50-70) patients showed a median OS of 6.4 months, and the CCTG study showed a median OS of 7.6 months (radiation alone) versus 9.3 months (radiation plus chemotherapy) 9,10. However, this regimen has a lower biological effective dose (BED) than the Stupp regimen, and patients in these cohorts had inferior outcomes when compared to elderly patients in the Stupp trial. Navarria et al. showed that elderly or frail patients (age $\geq 70$ years, Karnofsky performance scale (KPS) score $\leq 60$ ) treated with a dose of $52.5 \mathrm{~Gy}$ in 15 fractions (isoeffective to the Stupp regimen) had a median overall survival of 8 months, superior to the IAEA trial ${ }^{11}$. Other studies have also proven the efficacy of this fractionation regimen for this patient population ${ }^{12,13}$. Thus, we hypothesize that hypofractionated radiation therapy to $52.5 \mathrm{~Gy}$ in 15 fractions is a superior regimen to $40 \mathrm{~Gy}$ in 15 fractions for elderly or frail patients.

\section{Methods}

This retrospective study was approved by our institutional review board. At our $\mathrm{NCl}$-designated comprehensive cancer center, patients with GBM are eligible for hypofractionated radiation therapy consisting of 40 Gy or 52.5 Gy in 15 fractions if they are elderly (age $\geq 65$ ), frail (KPS $<70$ ), or at the discretion of the treating physician. Only newly diagnosed GBM patients were included in this study. Eligible patients were treated at our institution between 1/1/2010 and 10/30/2020. Diagnostic workup and radiation treatment for each patient were required to have been performed at this single cancer hospital.

Overall, 89 patients were identified based on a systematic query of patients with GBM who were treated with 15 fractions of radiation therapy. Reasons for exclusion in this study include the following: reirradiation of GBM, prior treatment for a non-GBM (examples include paraganglioma or low-grade glioma prior to transformation), grade III tumors, or not completing the prescribed treatment course. After evaluating patients for eligibility, 66 patients were included in the final analysis. Relevant demographic and clinical information was collected for each patient. ${ }^{14}$ 
For patients who received $52.5 \mathrm{~Gy}$, a $0.5 \mathrm{~cm}$ margin was added beyond the contrast enhancing area to create a clinical treatment boost volume (CTV $52.5 \mathrm{~Gy}$ ) with a $1 \mathrm{~cm}$ margin beyond FLAIR signal delineating the CTV $40 \mathrm{~Gy}$ volume. For patients receiving $40 \mathrm{~Gy}$, a $1.5 \mathrm{~cm}$ margin was delineated beyond the visible gross tumor volume (GTV) on MRI. A 3-5 mm planning treatment volume (PTV) was used for both groups. TMZ was offered to all patients in this study. None of the patients in this study received NovoTTF.

\section{Statistics}

Summary statistics were used to describe patients' demographics and clinical characteristics by radiation treatment regimen. Comparisons between treatment groups were tested using Pearson's Chi-square test, Fisher's exact test, and Wilcoxon's two-sample test. OS was defined as the time elapsed between surgery/biopsy and death from any cause, with censoring of patients who were lost to follow-up. Progression-free survival (PFS) was defined as the time elapsed between surgery/biopsy and progression. The Kaplan-Meier method was used to generate survival curves with corresponding log-rank tests for difference by stratification group. Lastly, we used complete-case Cox proportional hazards regression to estimate the adjusted hazard ratio (aHR) for treatment effect. Cox models adjusted for confounders (age and KPS) and 06 methylguanine DNA methyltransferase (MGMT) methylation status. A similar analysis was performed for progression free survival. Analyses were conducted in $\mathrm{R}$ version 4.0.5.

\section{Results}

Sixty-six patients were included in this analysis (Table 1). $41 \%$ of patients were treated with 52.5 Gy and $59 \%$ of patients were treated with $40 \mathrm{~Gy} .61 \%$ of patients in the 40 Gy arm and $57 \%$ of those in the 52.5 Gy arm demonstrated MGMT methylation. In total, only $63.6 \%$ of patients had a KPS $\geq 70$. The majority of patients (83.3\%) had surgical resection. Concurrent TMZ was prescribed in $88 \%$ of patients. There were no significant differences in measured patient characteristics between the two radiation treatment groups. 
Table 1

Patient characteristics stratified by radiation cohort

\begin{tabular}{|c|c|c|c|}
\hline Characteristic & $40 \mathrm{~Gy}, \mathrm{~N}=39^{1}$ & $52.5 \mathrm{~Gy}, \mathrm{~N}=27^{1}$ & p-value ${ }^{2}$ \\
\hline Sex & & & 0.2 \\
\hline Male & $20(51 \%)$ & $18(67 \%)$ & \\
\hline Female & 19 (49\%) & $9(33 \%)$ & \\
\hline Age at Diagnosis & $72(70,78)$ & $68(63,73)$ & 0.056 \\
\hline MGMT & & & 0.8 \\
\hline Unmethylated & 15 (39\%) & $9(43 \%)$ & \\
\hline Methylated & $23(61 \%)$ & 12 (57\%) & \\
\hline (N missing) & 1 & 6 & \\
\hline Multifocal & & & 0.4 \\
\hline No & $30(77 \%)$ & 23 (85\%) & \\
\hline Yes & $9(23 \%)$ & $4(15 \%)$ & \\
\hline Surgery & & & 0.2 \\
\hline No & $9(23 \%)$ & $2(7.4 \%)$ & \\
\hline Yes & $30(77 \%)$ & $25(93 \%)$ & \\
\hline Extent of Resection & & & 0.7 \\
\hline Subtotal & $21(54 \%)$ & $16(59 \%)$ & \\
\hline Gross total & $18(46 \%)$ & $11(41 \%)$ & \\
\hline PTV Volume (ccs) & $393(292,486)$ & $352(289,417)$ & 0.2 \\
\hline (N missing) & 0 & 6 & \\
\hline Concurrent Chemo & & & 0.13 \\
\hline No & $7(18 \%)$ & $1(3.7 \%)$ & \\
\hline Yes & 32 (82\%) & $26(96 \%)$ & \\
\hline KPS & & & $>0.9$ \\
\hline$<70$ & $13(34 \%)$ & $9(35 \%)$ & \\
\hline$\geq 70$ & $25(66 \%)$ & 17 (65\%) & \\
\hline (N missing) & 1 & 1 & \\
\hline
\end{tabular}


The median OS for the entire cohort was $11.2(95 \% \mathrm{Cl}: 8.5,14.2)$ months (Graph 1). Survival varied between radiation subgroups. Patients who received $52.5 \mathrm{~Gy}$ had a median OS of 14.1 months $(95 \% \mathrm{Cl}$ : $10.9, \mathrm{NA})$, while patients who received $40 \mathrm{~Gy}$ had a median OS of 7.9 months ( $95 \% \mathrm{Cl}: 6.3,14.2)$ representing a statistically significant difference in OS (aHR $0.38,95 \% \mathrm{Cl}: 0.18,0.80 ; p=0.011$ ). MGMT methylation status also demonstrated a significant role, with methylated and unmethylated patients having a median overall survival of 14.2 months $(95 \% \mathrm{Cl}: 9.3,22.4)$ and 8.8 months $(95 \% \mathrm{Cl}: 6.7,13.1)$, respectively (aHR $0.43,95 \% \mathrm{Cl} 0.22,0.82 ; \mathrm{p}=0.011)$.

The median PFS for the entire cohort was 6.0 months (95\% Cl: 4.7, 7.4) (Graph 2). PFS varied between radiation subgroups, with patients receiving 52.5 Gy having a median PFS of 7.5 months ( $95 \%$ Cl: 6.5, 12.4) and those receiving 40 Gy having a median PFS of 4.8 months ( $95 \% \mathrm{Cl}: 3.7,6.8)$. Methylated and unmethylated patients had a median progression free survival of 5.9 months ( $95 \% \mathrm{Cl}: 4.86,7.39)$ and 6.9 months (95\% Cl: $3.61,12.02)$, respectively (aHR $0.59,95 \% \mathrm{Cl}: 0.33,1.07 ; \mathrm{p}=0.083)$.

On univariable analysis, both methylation status (HR 0.53, 95\% Cl: $0.29,0.99 ; \mathrm{p}=0.045)$ and doseescalation (HR 0.47 [95\% Cl: 0.26 - 0.85]; $p=0.013$ ) were associated with improved OS. Increased planning target volume $(p=0.006)$ was associated with decreased OS. On multivariable analysis (Table 2$)$, both methylation status (aHR $0.43,95 \% \mathrm{Cl}: 0.22,0.85 ; \mathrm{p}=0.011$ ) and increased radiation dose (aHR $0.38,95 \%$ Cl: $0.18,0.80, p=0.011)$ remained independent prognostic factors for OS.

Table 2

Multivariable Cox Proportional Hazards Regression of Factors Associated with Overall Survival for $\mathrm{N}=$ 59 Patients

\begin{tabular}{|llll|}
\hline Characteristic & $\mathrm{HR}^{1}$ & $\mathbf{9 5 \%} \mathrm{Cl}^{1}$ & p-value \\
\hline Age at Diagnosis & 1.04 & $0.99,1.09$ & 0.13 \\
\hline Radiation Cohort & & & \\
\hline $40 \mathrm{~Gy}$ & - & - & \\
\hline $52.5 \mathrm{~Gy}$ & 0.38 & $0.18,0.80$ & $\mathbf{0 . 0 1 1}$ \\
\hline KPS & 0.99 & $0.96,1.02$ & 0.4 \\
\hline MGMT & & & \\
\hline Unmethylated & - & - & \\
\hline Methylated & 0.43 & $0.22,0.82$ & $\mathbf{0 . 0 1 1}$ \\
\hline
\end{tabular}

Increasing planning treatment volume $(\mathrm{p}=0.028)$ was associated with decreased PFS. In a multivariable model (Table 3), increased dose was associated with improved PFS, though no effect or decreased PFS was not contraindicated by the data (aHR $0.53,95 \% \mathrm{Cl}: 0.28,1.02 ; \mathrm{p}=0.057)$. 
Table 3

Multivariable Cox Proportional Hazards Regression of Factors Associated with Progression Free Survival for $\mathrm{N}=59$ Patients

\begin{tabular}{|llll|}
\hline Characteristic & HR$^{1}$ & $95 \% \mathrm{Cl}^{1}$ & p-value \\
\hline Age at Diagnosis & 1.01 & $0.97,1.05$ & 0.7 \\
\hline Radiation Cohort & & & \\
\hline 40 Gy & - & - & \\
\hline 52.5 Gy & 0.53 & $0.28,1.02$ & 0.057 \\
\hline KPS & 0.99 & $0.96,1.01$ & 0.2 \\
\hline MGMT & & & \\
\hline Unmethylated & - & - & \\
\hline Methylated & 0.59 & $0.33,1.07$ & 0.083 \\
\hline
\end{tabular}

When comparing the two radiation treatment groups, there was little evidence for differences in toxicity (Supplemental Table). There were two patients with Grade III toxicities in the $52.5 \mathrm{~Gy}$ group. One patient was hospitalized for multiple breakthrough seizures, although it should be noted that this patient had questionable compliance to a prescribed anticonvulsant medication. The second patient was hospitalized after experiencing two breakthrough seizures while on Keppra during the radiation treatment course. There were no Grade IV or V toxicities in the entire cohort.

\section{Discussion}

This is the first retrospective analysis of elderly and frail GBM patients that directly compares patients who received 52.5 vs. 40 Gy in 15 fractions. There were no significant differences in measured patient characteristics when comparing the two radiation groups. Within our cohort, OS survival was significantly improved in the 52.5 Gy group ( 14.1 months) when compared to the 40 Gy group (7.9 months). Increasing dose to 52.5 Gy was shown to be an independent prognostic factor for improved OS. There were no differences in toxicity between the two treatment groups.

This isoeffective treatment prescription has been studied prospectively. Navarria et al treated 30 patients in a single-arm phase II study to a total dose of $52.5 \mathrm{~Gy}$ in 15 fractions ${ }^{11}$. In this study, eligible patients were aged $\geq 70$ years old and had a KPS $\leq 60$, histologically confirmed glioblastoma, and had an estimated survival of $\geq 3$ months. The median OS survival in this study was 8 months. Scoccianti et al. treated patients in a single-arm phase II study to $52.5 \mathrm{~Gy}$ in 15 fractions with a simultaneous integrated boost to $67.5 \mathrm{~Gy}$ in 15 fractions ${ }^{13}$. Patients in this study belonged to Recursive Partitioning Analysis (RPA) class III (age $<50$, KPS 90-100) or IV (age $<50$, KPS $<90$, age $\geq 50$ ). There were seven elderly patients with a median OS of 11.6 months in this cohort ${ }^{13}$. These studies are the first to directly study 
isoeffective treatment dosing for elderly or frail patients; however, no study has directly compared $52.5 \mathrm{~Gy}$ vs. $40 \mathrm{~Gy}$ in 15 fractions.

It is notable that patients who received $52.5 \mathrm{~Gy}$ in our cohort had superior outcomes when compared to historical hypofractionation studies for elderly patients or those with a poor performance status. The Minnitti study ( $79 \%$ of patients had a KPS $\geq 70$ ) demonstrated a median OS of 9.3 months for patients $>70$ years old treated with 30 Gy in 6 fractions over two weeks ${ }^{15}$. The Nordic trial ( $80 \%$ of patients with a WHO performance status of 0-1) demonstrated a median OS of 7.0-8.8 months for patients treated with hypofractionated radiation therapy of $34 \mathrm{~Gy}$ in 10 fractions over two weeks ${ }^{16}$. The CCTG trial $(77 \%$ of patients had an ECOG of 0-1) demonstrated a median OS of 7.6-9.3 months for patients treated with 40 Gy in 15 daily fractions ${ }^{10}$. In our cohort, $62.9 \%$ of patients who received $52.5 \mathrm{~Gy}$ had a KPS $\geq 70$, and $64.1 \%$ of patients who received $40 \mathrm{~Gy}$ had a KPS $\geq 70$. The patients who received $40 \mathrm{~Gy}$ in our cohort had a comparable median OS (7.9 months) to the Minnitti, Nordic, and CCTG studies (median OS from 7.0-9.3 months). Meanwhile, our 52.5 Gy group had a superior median OS of 14.1 months.

These data suggest that elderly or frail patients are potentially receiving a sub-therapeutic radiation dose with the current standard-of-care hypofractionated treatment. Walker et al. showed that increasing the dose from $\leq 45 \mathrm{~Gy}$ to $50 \mathrm{~Gy}, 55 \mathrm{~Gy}$ and $60 \mathrm{~Gy}$ in 1.8-2.0 Gy fractions improved the median overall survival from 13.5 weeks to 28 weeks, 36 weeks and 42 weeks, respectively ${ }^{4}$. This study guided the radiation prescription for the seminal Stupp trial to be $60 \mathrm{~Gy}$ in 30 fractions which demonstrated a median OS of 10.9-11.8 months for elderly patients. However, the IAEA and the CCTG studies used prescriptions with a lower BED in comparison to Stupp dosing. Our retrospective cohort showed superior outcomes in favor of $52.5 \mathrm{~Gy}$ when compared to the $40 \mathrm{~Gy}$ group. This is likely because $52.5 \mathrm{~Gy}$ in 15 fractions has a BED that is isoeffective to Stupp dosing (BED = $75 \mathrm{~Gy}$ ) as opposed to that of $40 \mathrm{~Gy}$ in 15 fractions (BED = 53.35 Gy).

The greatest strength of this study is that there has never been a direct comparison between $52.5 \mathrm{~Gy}$ and 40 Gy given in 15 fractions for elderly or frail patients. This study included patients with both a good and poor performance status. We were able to show that a hypofractionated radiation course with an isoeffective BED to $60 \mathrm{~Gy}$ in 30 fractions was effective and did not lead to increased toxicity. Additionally, this radiation prescription does not increase the treatment burden for elderly or frail patients because it requires the same number of treatment visits when compared to the IAEA and CCTG studies. Limitations of this study include a relatively small sample size of 66 patients. Information about neurocognitive function was not collected for this study. An additional limitation is this study's retrospective nature. A large, randomized controlled trial is needed to validate these results.

\section{Conclusion.}

We hypothesize that the de-escalation of radiation therapy that has occurred for elderly or frail patients has negatively impacted survival for this patient population. We propose a fractionation scheme that is 
isoeffective to Stupp dosing and leads to improved OS by up to 6 months when compared to prospective hypofractionation cohorts. This is a safe and effective regimen that requires prospective validation.

\section{Declarations}

Conflict of Interest:All authors declare no conflict of interest related to this manuscript.

Informed Consent: Since this study is retrospective, informed consent was not obtained in this study.

Ethical Approval: All procedures performed in studies involving human participants were in accordance with the ethical standards of the institutional and/or national research committee and with the 1964 Helsinki declaration and its later amendments or comparable ethical standards.

Funding: No funding was received to assist with the preparation of this manuscript.

\section{References}

1. Wen PY, Kesari S. Malignant Gliomas in Adults. New England Journal of Medicine. 2008; 359(5):492507.

2. Walker MD, Alexander E, Jr., Hunt WE, et al. Evaluation of $B C N U$ and/or radiotherapy in the treatment of anaplastic gliomas. A cooperative clinical trial. Journal of neurosurgery. 1978; 49(3):333-343.

3. Kristiansen K, Hagen S, Kollevold T, et al. Combined modality therapy of operated astrocytomas grade III and IV. Confirmation of the value of postoperative irradiation and lack of potentiation of bleomycin on survival time: A prospective multicenter trial of the scandinavian glioblastoma study group. Cancer. 1981; 47(4):649-652.

4. Walker MD, Strike TA, Sheline GE. An analysis of dose-effect relationship in the radiotherapy of malignant gliomas. International Journal of Radiation Oncology, Biology, Physics. 1979; 5(10):17251731.

5. Stupp R, Hegi ME, Mason WP, et al. Effects of radiotherapy with concomitant and adjuvant temozolomide versus radiotherapy alone on survival in glioblastoma in a randomised phase III study: 5-year analysis of the EORTC-NCIC trial. Lancet Oncology. 2009; 10(5):459-466.

6. Ostrom QT, Gittleman H, Farah P, et al. CBTRUS statistical report: Primary brain and central nervous system tumors diagnosed in the United States in 2006-2010. Neuro-oncology. 2013; 15 Suppl 2:ii1ii56.

7. Keime-Guibert F, Chinot O, Taillandier L, et al. Radiotherapy for glioblastoma in the elderly. New England Journal of Medicine. 2007; 256(15):1527-1535.

8. Roa W, Brasher PMA, Bauman G, et al. Abbreviated Course of Radiation Therapy in Older Patients With Glioblastoma Multiforme: A Prospective Randomized Clinical Trial. Journal of Clinical Oncology. 2004; 22(9):1583-1588. 
9. Roa W, Kepka L, Kumar N. International Atomic Energy Agency Randomized Phase III Study of Radiation Therapy in Elderly and/or Frail Patients With Newly Diagnosed Glioblastoma Multiforme. Journal of Clinical Oncology. 2015; 33(35):4145-4150.

10. Perry JR, Laperriere N, Christopher O, Alba B. Short-Course Radiation plus Temozolomide in Elderly Patients with Glioblastoma. New England Journal of Medicine. 2017; 376(11):1027-1037.

11. Navarria P, Pessina F, Cozzi L, et al. Phase II study of hypofractionated radiation therapy in elderly patients with newly diagnosed glioblastoma with poor prognosis. Tumori Journal. 2019; 105(1):47-.

12. Ammirati M, Chotai S, Newton H, Lamki T, Wei L, Grecula J. Hypofractionated intensity modulated radiotherapy with temozolomide in newly diagnosed glioblastoma multiforme. Journal of Clinical Neuroscience. 2014; 21(4):633-637.

13. Scoccianti S, Krengli M, Marrazzo L, et al. Hypofractionated radiotherapy with simultaneous integrated boost (SIB) plus temozolomide in good prognosis patients with glioblastoma: a multicenter phase II study by the Brain Study Group of the Italian Association of Radiation Oncology (AIRO). La Radiologia Medica. 2018(1):48.

14. Brown AP, Barney CL, Grosshans DR, et al. Proton Beam Craniospinal Irradiation Reduces Acute Toxicity for Adults With Medulloblastoma. International Journal of Radiation Oncology, Biology, Physics. 2013; 86(2):277-284.

15. Minniti G, Sanctis V, Muni R, et al. Hypofractionated radiotherapy followed by adjuvant chemotherapy with temozolomide in elderly patients with glioblastoma. Journal of Neuro-Oncology. 2009; 91(1):95.

16. Malmström A, Grønberg BH, Marosi C, et al. Temozolomide versus standard 6-week radiotherapy versus hypofractionated radiotherapy in patients older than 60 years with glioblastoma: the Nordic randomised, phase 3 trial. Lancet Oncology. 2012; 13(9):916-926.

\section{Figures}



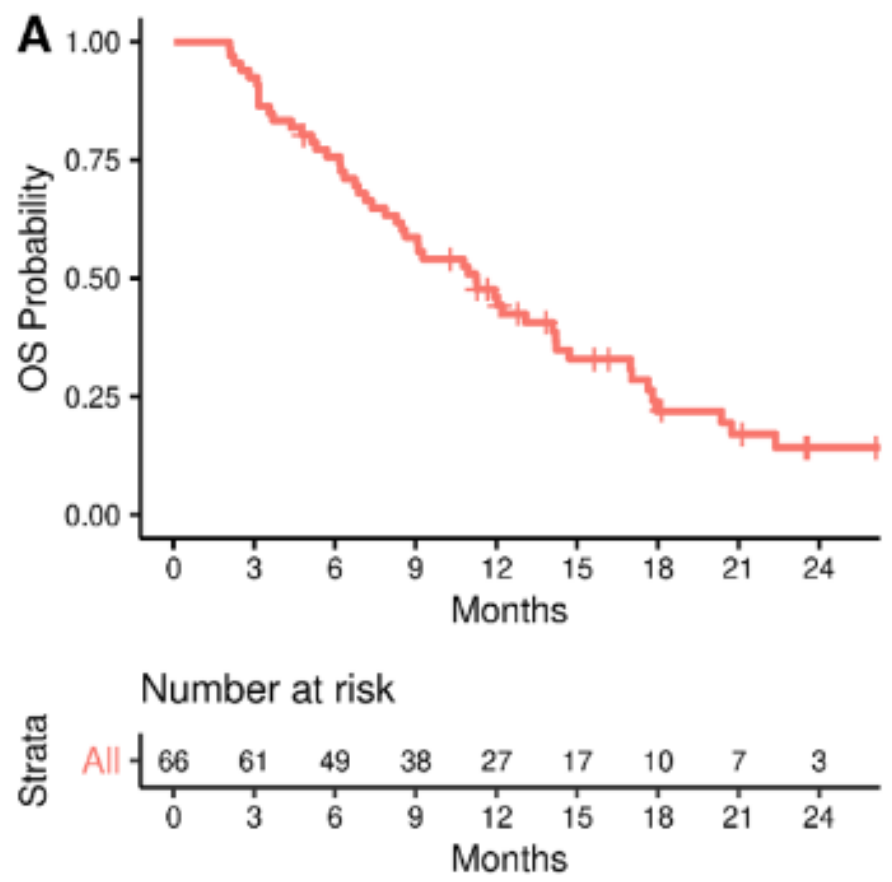

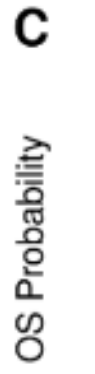
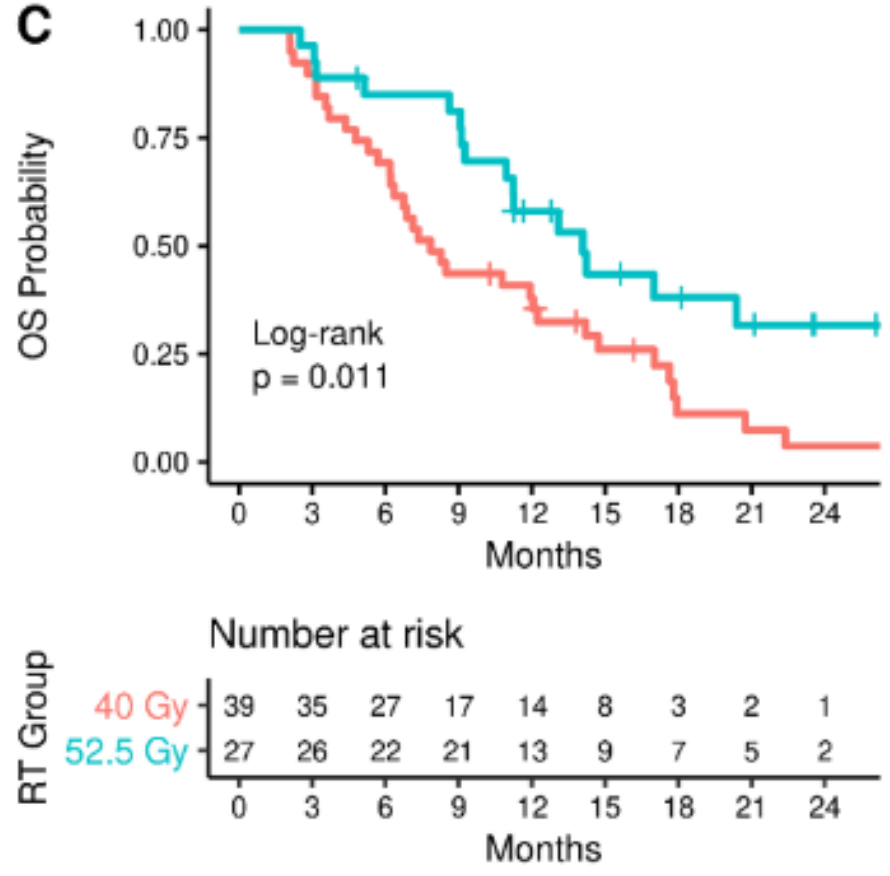

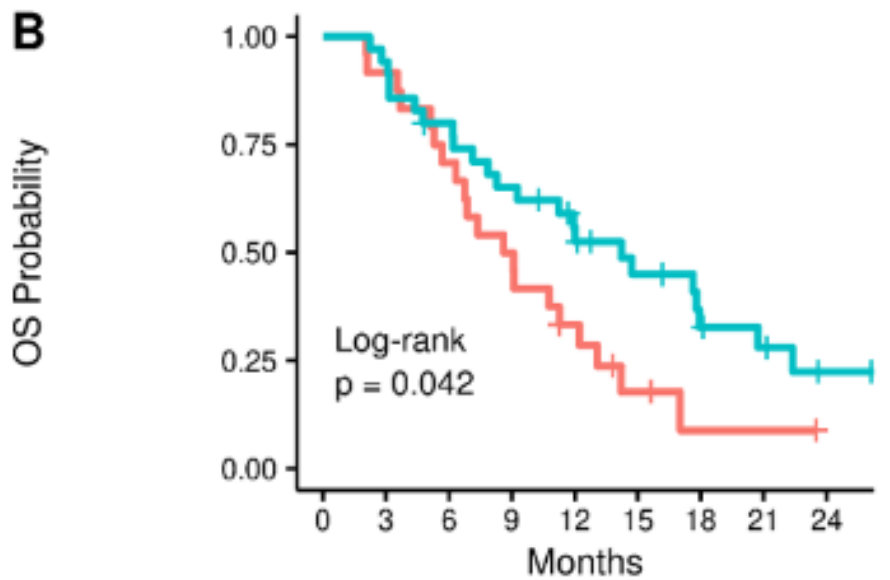

Number at risk
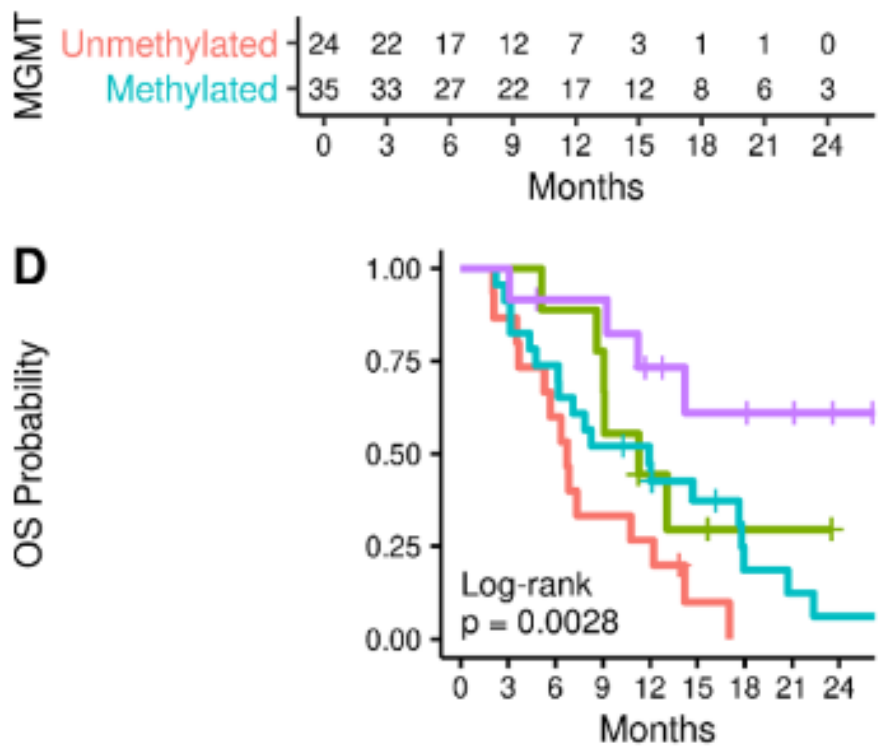

Number at risk

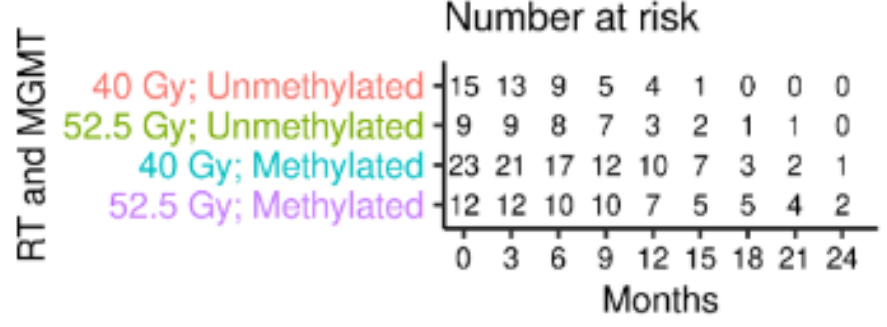

\section{Figure 1}

Kaplan-Meier curves evaluating overall survival. (A) OS for entire cohort. (B) OS stratified by MGMT methylation status. (C) OS stratified by radiation dose. (D) OS stratified by prognostic group. Abbreviations: OS - overall survival, KPS, Karnofsky performance status. 

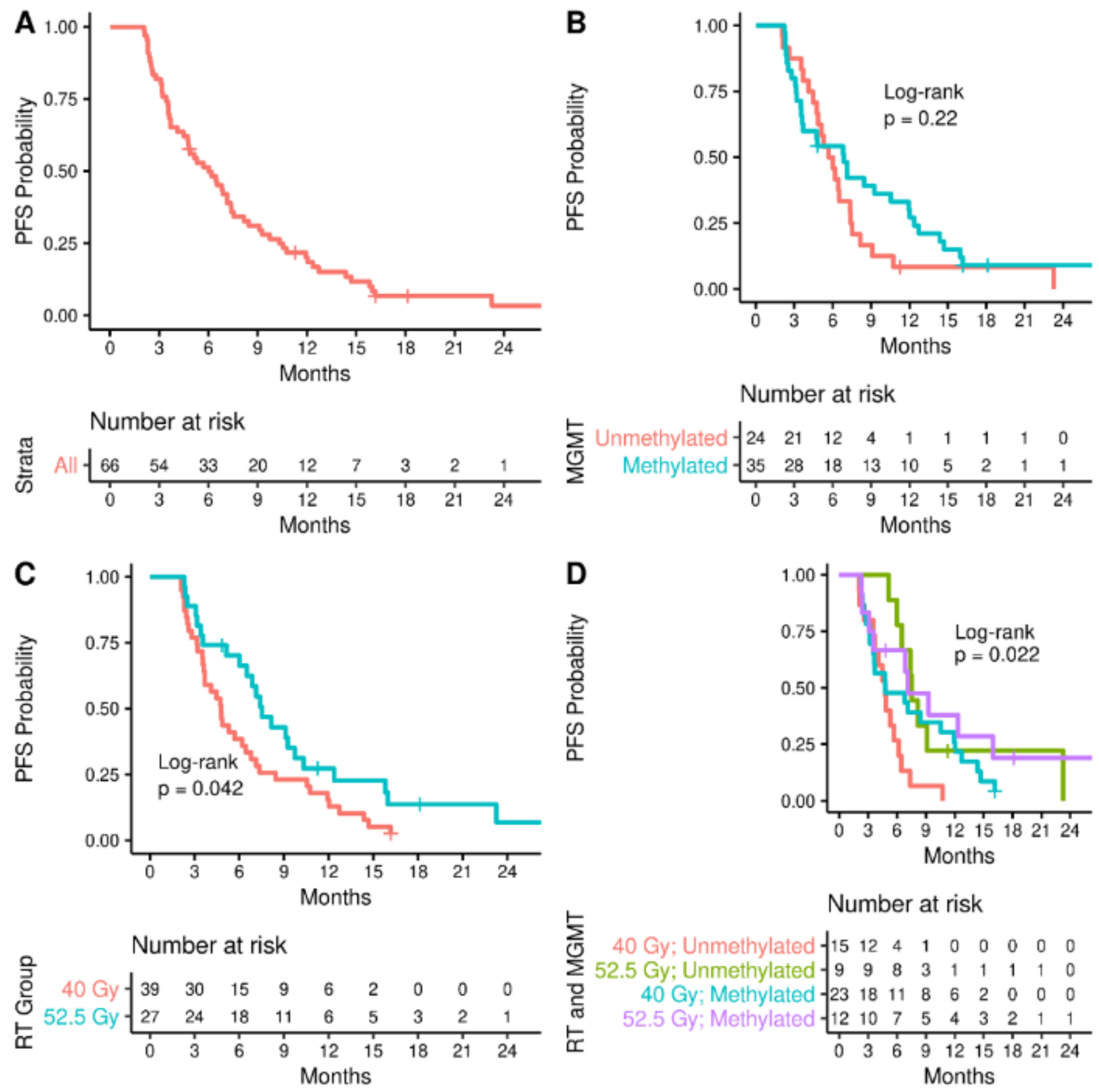

Figure 2

Kaplan-Meier curves evaluating progression free survival. (A) PFS for entire cohort. (B) PFS stratified by MGMT methylation status. (C) PFS stratified by radiation dose. (D). PFS stratified by prognostic group. Abbreviations: PFS - progression free survival, KPS, Karnofsky performance status.

\section{Supplementary Files}

This is a list of supplementary files associated with this preprint. Click to download. 
- SupplementalTableOSUJNO10182021.docx

Page 14/14 